\title{
Role of miRNA Signatures in Health and Productivity of Livestock
}

\author{
Aamir Bashir Wara ${ }^{1}$, Supriya Chhotaray ${ }^{1}$, Burhan Ud Din Shafi ${ }^{2}$, Snehasmita Panda ${ }^{1}$, \\ Mitek Tarang ${ }^{1}$, Saleem Yosuf ${ }^{3}$, Naseer Ahmad Baba ${ }^{3}$ and Amit Kumar ${ }^{1}$ \\ ${ }^{1}$ Division of Animal Genetics, ${ }^{2}$ Division of Animal Nutrition, \\ ${ }^{3}$ Division of Animal Genetics, ICAR- National Dairy Research Institute, \\ Karnal, 132001, India \\ *Corresponding author
}

\section{A B S T R A C T}

\begin{tabular}{|l|l|l}
\hline Keywords & $\begin{array}{l}\text { The knowledge and implementation of microRNA expression profiles in disease diagnosis } \\
\text { and target gene identification have grown exponentially. MicroRNAs as markers of } \\
\text { MicroRNA, } \\
\text { Livestock, and } \\
\text { Biomarker }\end{array}$ & $\begin{array}{l}\text { explored in human and animals. Several target gene sites have been identified } \\
\text { experimentally and some are predicted computationally in some livestock and pet species, } \\
\text { and polymorphism at these sites is viewed as a basis for selection. While in the medical } \\
\text { sciences, use of miRNA as a biomarker has gained impetus, the accomplishment in animal } \\
\text { science remains still below adequacy. Below we review the history of its discovery in } \\
\text { various healthy and diseased tissues and body fluids that could pave a way for } \\
\text { improvement of health and productivity in animals. }\end{array}$ \\
\hline $\begin{array}{l}\text { Accepted: } \\
\text { Available Online: }\end{array}$ April 2019 &
\end{tabular}

\section{Introduction}

Eukaryotic gene regulation is known to occur mostly at the transcriptional level. Recent research has demonstrated that post transcriptional mechanisms also play important role in regulating the expression of eukaryotic genes. Some of these involve, base-pairing of small, non-coding RNAs with target sequences in messenger RNA molecules, thereby interfering with gene expression. While some of these non-coding transcripts are long (long non-coding RNA) such as Xist, some are short (short non-coding RNA or sncRNA) such as microRNAs
(miRNA), small interfering RNAs (siRNA) and piwi interacting RNAs (piRNAs).

Micro RNAs are 21-28 base pairs long, small non-coding RNAs found associated with gene expression. More than 60\% of genes undergo direct miRNA regulation and they regulate gene expression in several cellular processes such as signal transduction, cell cycle, differentiation, and transformation (Friedman et al., 2009). In different livestock species, miRNAs have been found to have role in regulating the expression of proteincoding genes involving different physiological and pathological processes. 
miRNA expression profiles in livestock species are mostly concerned with productivity, fertility, embryo development, and disease resistance. miRNAs are also known to play a role of biomarkers in certain disease diagnosis. Polymorphisms at miRNA binding sites of target genes can lead a way for genetic improvement through associating it with phenotypes of interest during the process of genomic selection in livestock breeding programs (Fatima and Morris, 2013). Till date miRNA expression profiles have been developed across a variety of tissues from livestock species. Existence of miRNAs has also been reported in body fluids like milk and blood where they exist in microvesicles or exosomes (Fatima and Morris, 2013). miRNAs, which play vital role in biological processes in all cell types across body, are reported to exhibit ubiquitous expression (Jin et al., 2009).

Micro RNAs are synthesized as nascent longer miRNA transcript, i.e., pri-miRNA in the nucleus and then processed by the enzyme Drosha to form Pre-miRNA. Pre-miRNA is exported to the cytoplasm with the help of exportin 5 protein, and then cleaved by an RNAseIII enzyme (Dicer) to form mature miRNA. RNA induced silencing complex (RISC) then binds to one of the strands of mature miRNA. The complementary sequence of RISC incorporated miRNA base pairs with target mRNA leading to mRNA degradation. The overall pathway of miRNA synthesis is presented in Figure 1.

Aberrant expression of miRNAs affects the regulation of many cellular functions and gene networks. The detection of a small number of miRNAs provides important information about the course, stage and prognosis of the disease (Wang et al., 2015). Tissue and body fluid specific expression of miRNAs such as expression in skeletal muscles of sheep, goat and pig while expression in mammary gland and milk of cattle, provides a guide to select desirable phenotypes of economic importance. However, a voluminous number of miRNA studies are conducted on humans as compared to studies in animals. So, in the present study we review regarding the techniques of miRNA detection and application of their expression profiles in studies of disease diagnosis, productivity and fertility traits in various domestic animal species.

\section{miRNA detection methods}

Precise miRNA detection in clinical samples is the key to successful miRNA guided diagnostics. There are several methods developed to date for miRNA detection. These include small RNA sequencing (Hafner et al., 2012), quantitative real-time PCR (qRT-PCR) (Chen et al., 2005), miRNA microarray (Castoldi et al., 2007), multiplexed miRNA detection with color coded probe pairs (Geiss et al., 2008), and miRNA in situ hybridization (Nelson et al., 2006). Below, we enlist some of the common approaches reviewed by Gustafson et al., (2016) to detect miRNA keeping in view the diagnostic need. The clinical testing should be rapid, sensitive, accurate, labour nonintensive, easy to analyse, and cost-effective.

\section{Small RNA sequencing}

Quantitative detection along with comprehensive profiling is its key features. It has high sensitivity and specificity but requires intensive labour and cost.

\section{Quantitative real-time PCR}

It is the easiest way of amplification based detection of miRNA and is comparatively inexpensive and clinically tractable with high sensitivity and specificity. It is mostly used to quantify the levels of a defined set of miRNAs thus has limited profiling. 


\section{miRNA microarray}

It is a hybridization based technique that provides comprehensive profiling and can simultaneously measure large number of circulating miRNA. It has low sensitivity and specificity. It is unable to detect novel unannotated miRNA and is expensive.

\section{Nano stringn counter expression system}

It is an emerging technique based on hybridisation that produces a quantitative profile of miRNA. It is highly multiplexed, with high sensitivity and specificity and provides direct digital detection. High cost incurring in comparison to other methods.

\section{Northern blotting}

It is the most widely used method but has low sensitivity (de Planell-Saguer and Rodicio, 2011).

\section{Bioluminescence miRNA detection}

Solid-phase Protein complementation driven by DNA hybridization that provides direct detection of miRNA in total RNA from tissue and cells. It is a high-throughput miRNA profiling technique that is highly sensitive and rapid (Cissell et al., 2008).

\section{Surface enhanced Raman spectroscopy (SERS)}

It involves the mechanism of absorption of miRNA to silver or gold nano-rods for labelfree miRNA detection. It has excellent reproducibility and single nucleotide specificity but requires high analytical skill (Driskell et al., 2008).

\section{Surface plasmon resonance imaging (SPRI)}

Is a label-free detection method that can monitor molecular interactions on a surface.
A combination of surface poly-(A) enzyme chemistry and gold nanoparticle-amplified SPRI measurements is used to detect multiple miRNAs at attomole levels (Fang et al., 2006). Is has excellent specificity but cumbersome analytical procedures are involved.

\section{Role of mirna in livestock species}

There are several studies that demonstrate a correlation between expression profiles of circulating miRNAs and various bacterial (Paratuberculosis), viral (foot and mouth disease, bovine virus diarrhoea), parasitic (Echinococcosis), and metabolic (mastitis) diseases. Still, in livestock, studies of miRNA expression profiles are predominantly concerned with their association with traits of economic importance, especially traits associated with milk, meat, and egg production and traits influencing animal productivity and fertility. So, below we brief the role of circulating miRNA in different species.

\section{Cattle}

One of the most economically important disease that causes a huge loss is Foot-andmouth disease. Stenfeldt et al., (2017) observed that, miR-1281 was significantly reduced at both acute and persistent infection stages while bta-miR-17-5p was expressed in the highest level at acute infection stage.

They also reported that bta-miR-31 level was significantly increased during persistence stage. Gutkoska et al., (2017) reported the role of host miR-203a in FMDV replication in host, where miR-203a-3p and miR-203a-5p showed anti-viral activity for FMDV by regulating Sam68 and Survivin expression in host. Basagoudanavar et al., (2018) reported 72 up-regulation of 72 on 2 DPI, among which, 39 miRNAs were statistically differentially upregulated. They also reported 
two fold change in expression of bta-miR-215p, bta-miR-101, bta-miR-126-3p, bta-miR145 , bta-miR-197 and bta-miR-223 in serum samples in 2 DPI.

Taxis et al., (2017) investigated miRNA profiles of Bovine viral diarrhoea virus (BVDV) infected colostrum deprived male Holstein calves. They observed differential expression of two circulating miRNAs (BtamiR-423-5p and Bta-miR-151-3p) in acute stage of fever and lymphopenia. However, only Bta-miR-151-3p expression was significantly up-regulated at 9 days postinfection compared to the control group. Thus, Dong et al., (2017) suggested that these two miRNAs based on the above findings may not serve as appropriate diagnostic biomarkers.

Johne's disease also known as Paratuberculosis is a chronic disease caused by Mycobacterium paratuberculosis. Shaughnessy et al., (2017) reported that there is no significant differential expression of circulating miRNA in Paratuberculosis infection. Recently, Malvisi et al., (2016) found significant down-regulation of bta-mir19b, bta-mir-19b-2, bta-mir-1271, bta-mir100, bta-mir-301a, bta-mir-32, and one Novel miRNA, while bta-mir-6517 and bta-mir7857 levels were increased in positive animals.

\section{Mastitis and mammary tissue infections}

Bovine mastitis, defined as inflammation of the mammary gland', can have an infectious or non-infectious aetiology. Organisms as diverse as bacteria, mycoplasma, yeasts and algae have been implicated as causes of the disease. Watts (1988) identified 137 different organisms as a cause of mastitis. Mastitis remains a major challenge to the worldwide dairy industry despite the widespread implementation of mastitis control strategies. Escherichia coli and Streptococcus uberis are now the two most common causes of bovine mastitis.

Ogorevc et al., (2009) reported 359 putative target sites for miRNAs on a set of candidate gene and genetic markers for mastitis and milk production in mammary gland. They suggested the role of miR-31during early lactation period which is increased compared with the dry period. It was also reported by them that DQA2-SV1 as well as miR-296, miR-2430, and miR-671 had a higher expression, while miR-2318 had a lower expression in tissue affected by mastitis. The expression of specific miRNAs in response to bacterial infection can be examined by in vivo challenge of bacterial pathogens into mammary tissues of the cows, for example, Streptococcus uberis (Lawless et al., 2013; Lawless et al., 2014; Naeem et al., 2012), Staphylococcus aureus (Jin et al., 2014) and Escherichia coli (Dilda et al., 2012; Jin et al., 2014). In Streptococcus uberis infection of mammary tissue it was observed that, miR181, miR-16, and miR-31 had a lower expression in infected tissues, while miR-223 had an increased expression (Naeem et al., 2012). miR-31 and miR-223 were found to be involved in inhibition of lipid metabolism, whereas miR-181a plays major roles in phagocytosis and antigen processing and presentation in infected mammary tissue (Naeem et al., 2012).

Staphylococcus aureus is another important pathogen causing mastitis in cattle, leading to substantial milk, quality, and economic loss to dairy farmers worldwide. Sun et al., (2015) reported up-regulation in miRNA expression profiles of bta-miR-142-5p and bta-miR-223, during mid-lactation prior to and after infection (48 h) in Holstein cows. The inflammatory processes in mammary glands stimulated by E. coli LPS and S. aureus enterotoxin B (SEB)in bovine monocytes (CD14+ cells) may possibly be associated 
with miR-9, miR-125b, miR-155, miR-146a and miR-223 (Dilda et al., 2012). In particular, bta-miR-142-5p, and miR-223 are potential biomarkers for early detection of bacterial infection of the mammary gland.

Many researchers have also emphasized the role of miRNA in bovine fertility. In one study maturation of oocytes at different stages were studied and seven miRNAs (miR-496, miR-297, miR-292-3p, miR-99a, miR-410, miR-145, and miR-515-5p) were reported to be more abundant in mature oocytes, while two miRNAs (miR-512-5p and miR-214) were more abundant in immatures (Tesfaye $e t$ al., 2009). In another study, seven miRNAs were reported to be differentially expressed in the spermatozoa of high- and low-fertile Holstein bulls (Govindaraju et al., 2012).

miRNAs also play an important role in pregnancy diagnosis and can serve as the most potent biomarker for early pregnancy detection. Ioannidis and Donadeu (2017) confirmed significant differences in the expression of let-7f, let-7c, miR-30c, miR101, miR-26a, miR-205 and miR-143 between $0-60^{\text {th }}$ days of pregnancy. Significant up-regulation of circulatory levels of miR-26a as early as $8^{\text {th }}$ (Ioannidis and Donadeu, 2017) and $30^{\text {th }}$ day (Markkandan et al., 2018) of conception has been observed. Some miRNAs (miR-92a and miR-486) were found to be highly expressed post-pregnancy (Markkandan et al., 2018).

\section{Sheep and goat}

The most common disease affecting small ruminants is Peste des Petits Ruminants (PPR) that causes a great economic loss. Qi et al., (2018) in an experiment, infected peripheral blood mononuclear cells (PBMC) of goats with Nigeria 75/1 vaccine virus (a common vaccine used for PPRV) and studied the expression profiles of miRNA in mock infected PBMC and PPRV infected PBMC. They found a total of 316 miRNA (103 known and 213 novel miRNA) those were differentially expressed in the two groups. They also found chi-miR-204-3p and one novel miRNA that were up-regulated whereas chi-miR-338-3p, chi-miR-30b-3p, chi-miR199a-5p, chi-miR-199a-3p, chi-miR-1, and two novel miRNAs are down-regulated in mock-infected goat PBMC compared with the PPRV-infected cells. Pandey et al., (2017) identified a total of 67 and 37 DEmiRNAs in lungs and 50 and 56 DEmiRNAs in spleen of PPR infected goats and sheep, respectively. They also suggested a total of 20 and 11 miRNAs are common in expression in both sheep and goat PPRV infected spleen and lung, respectively.

In small ruminants like sheep and goat the muscle traits are of chief economic importance. One of the gene regulating skeletal muscle growth is myostatin and SNPs at binding sites formiR-1 and miR-26 on the myostatin locus have been linked with muscular hypertrophy in sheep (Clop et al., 2006). The Callipygian (CLPG) phenotype is a paternally inherited muscular hypertrophy in Texel sheep and Caiment et al., (2010) reported the expression of some miRNAs those were associated with CLPG phenotype. In a comparative study between Ujumqin and Texel lamb longissimus muscle, miR-1 and miR-214 were reported to be overexpressed in Ujumqin lambs (Zhang et al., 2013).

In the mammary gland of Prealpes-duSudewes'miR-21 and miR-205were expressed in early and mid-pregnancy with miR-21 being more highly expressed in epithelial cells in early pregnancy and miR-205 more highly expressed during late pregnancy (Galio et al., 2013). In addition, they also reported the increased expression of miR-200a, miR200b, miR-200c, miR-141, and miR-429 in late pregnancy and lactation. 
Li et al., (2011) have identified a total of 346 conserved and 95 novel miRNAs in dairy goats' mammary gland at peak lactation and dry period. In another study of Laoshan Dairy goats, miRNAs expression profile showed upregulation of miR-17a and down-regulation of miR-883 in late lactation in the mammary tissue (Ji et al., 2012).In both the periods, miR-143,miR-143-3p, miR-148a-3p, let-7-5p, and let-7b were co-expressed.

\section{Pig}

The porcine whipworm infestation that occurs due to Trichuris suis, is epidemic all over the world, leads to reduced feed efficiency, reduced growth rates, haemorrhagic diarrhoea, and death (Roepstorff et al., 2011). Hansen et al., (2016) found significant upregulation in levels of circulating miRNA, ssc-let-7d-3p, at 8 weeks post infection. But this miRNA may not be a suitable biomarker as the pre-patent period of $T$. suis is 6-7 weeks.

In pigs meat quality traits are of much importance due to upsurge in pork consumption. In porcine skeletal muscle miR1 was found to be moderately expressed in developmental stages while highly expressed in adult tissue (McDaneld et al., 2009). In a study by Nielsen et al., (2010), miR-1and miR-206 were found to be highly expressed in porcine skeletal muscle. In one study, Holley et al., (2011) found polymorphisms inmiR-1 from three breeds of pig that affect muscle fibre formation, while Cho et al., (2010) reported that miR-1 andmiR-133 were more highly expressed in porcine skeletal muscle compared with adipose tissues. The fat content of meat important to maintain its texture, flavour and quality. In a comparative study of miRNA expression between back fat of Large White (lean type) and Meishan (Chinese indigenous) pigs, Chen et al., (2012) observed increased expression of miR-215, miR-135, miR-224, and miR-146b in Large
White pigs, whereas miR-1a, miR-133a, miR122, miR-204, and miR-183 in Meishan pigs. Expression of miRNA varies from breed to breed and also differentially expressed in tissues for example the miRNA expressed in adipose tissues may not be expressed in skeletal tissues. Li et al., (2012), in a study reported increased expression of miR-296 in adipose compared with muscle tissues in the Landrace breed, whereas, the Lantang breed showed higher expression of miR-17 in adipose compared with longissimus muscle. They also reported higher expression of miR143 in adipose tissue than in muscle tissue in both the breeds.

The genetically modified pigs serve as the most suitable source of organ for xenotransplantation in humans due to longer survival and less rejection (Cooper et al., 2016). To diagnose the cases of acute rejection (AR), disease recurrence, and drug toxicity after xenotransplantation the recommended practice is biopsy. Shan et al., (2011), stated the possible role of miRNA in detecting allograft function or acute rejection. Levels of miR-142-5p, miR-155 and miR-223 individually can predict acute rejection with $>90 \%$ sensitivity and specificity in human renal allografts (Tao et al., 2015).

\section{Role of mirna in pet animals}

\section{Canines and felines}

Canines are most susceptible to cardiovascular diseases and there are ample of researches regarding role of miRNA in these diseases in different breeds of dogs. miRNA play an important role in the development of heart failure and cardiac remodelling (Condorelli et al., 2014). Changes in the expression of miR-1, miR- 21, miR-23, miR133, miR-199, miR-208 and miR-320 in Congestive heart failure (CHF) has been reported by Topkara and Mann (2011). It also plays an important role in mitral valve disease 
in dogs. Li et al., (2012) reported underexpression of microRNAs; cfa-miR-487b and cfa-miR-582 and overexpression of cfa-miR103, cfa-miR-98, cfa-let-7c and cfa-let-7b in symptomatic mitral valve disease. Hulanickaet al., (2014) studied miRNA expression in Dachshunds in various phases of MVD. They reported down-regulation of miR-30b in dogs with class $B$ and $C$ disease when compared to those with class $\mathrm{A}$, while the microRNAs miR-133b, miR-125, miR126, miR-21, miR-29b and miR-30b were down-regulated in dogs with class $\mathrm{C}$ disease. They suggested the role of $\mathrm{miR}-133 \mathrm{~b}$ as a potential biomarker for stage $\mathrm{C}$ congestive heart failure. A common type of arrhythmia in dogs is Atrial Fibrillation (AF). Zhang et al., (2015) reported over-expression of micro-cfamiR-206 in dogs with AF. They also reported dysregulated expression of 16 other microRNAs in dogs with AF. Li et al., (2012) showed down-regulation of miR-133 and miR-30 in dogs with atrial fibrillation.

\section{Feline cardiomyopathy}

The most common type of feline cardio myopathy is Hypertrophic cardiomyopathy (HCM) that is known to have a strong genetic predisposing factor. According to Weber et al., (2015), 49 microRNAs are related to cardiac hypertrophy in cats. miR-381-3p, miR-486-3p, miR5700, miR-513a-3p and miR-320E has already been recognized to be up-regulated in cats with stable congestive heart failure secondary to HCM.

Other than cardiovascular diseases, a recent study by Dirksen et al., (2016) reported the role of miR-122, as a biomarker to measure hepatocyte damage in Labrador Retriever dogs. Fujiwara-Igarashi et al., (2015) assessed 277 microRNAs out of which let-7b, miR223, miR-25 and miR-92a showed decreased expression while miR-423A showed overexpression in dogs with lymphoma compared with the control group.

Fig.1 miRNA biogenesis and function in gene expression regulation

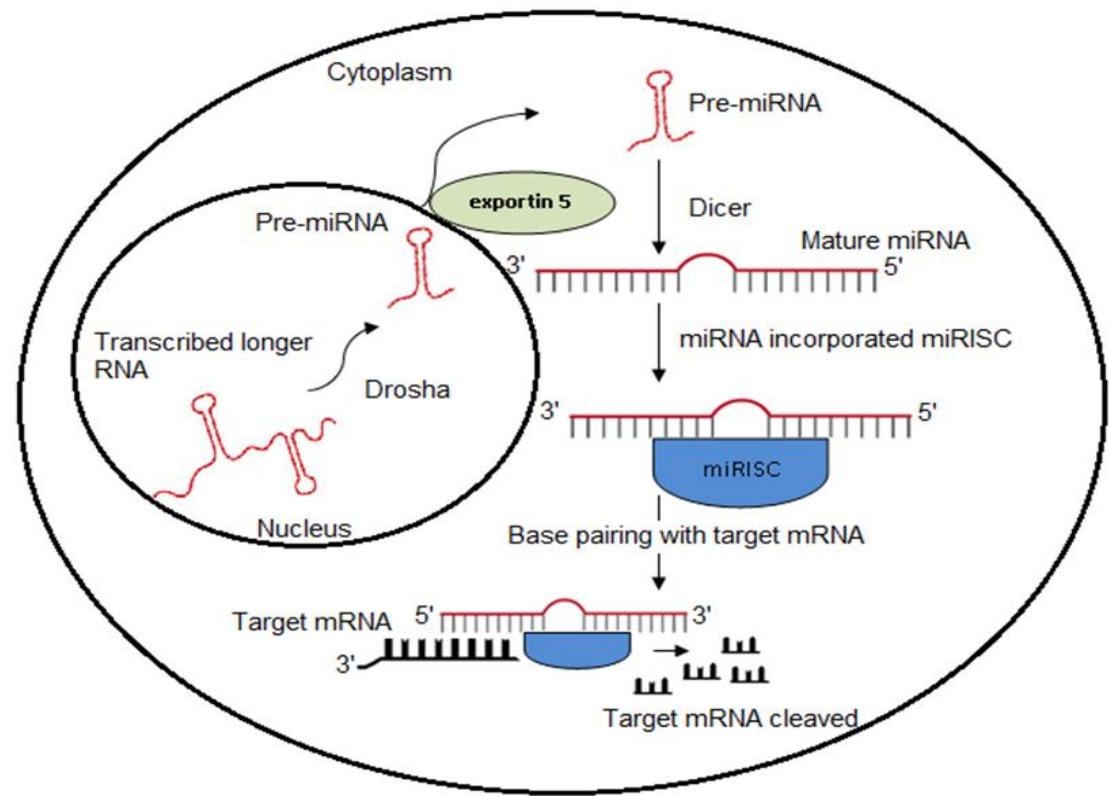




\section{Parvo-viral infection}

Feline parvovirus (FPV) and canine parvovirus (CPV) variants are one of the most common pathogens of feline and canine species. Zhou et al., (2017) reported differential expression of miRNA in cats and dogs infected with FPV and CPV. 156 miRNAs showed up-regulation and 87 showed down-regulation in the FPV infection group, while 91 were up-regulated and 31 were downregulated in the CPV infection group. In common, 10 were up-regulated and 26 down-regulated in both the FPV and CPV infection groups.

In conclusion we have highlighted the role of miRNAs in regulation of important economic traits and diseases in livestock animals in this review. Several studies have shown that miRNAs are differentially expressed in different tissues and body fluids potentially making them useful for associating them with various traits. Many researchers have studied miRNA signatures that are representative of diseases, including cancer, viral and bacterial infections, and fertility disorders.

Differential expression of miRNAs in diseased tissue makes it an important biomarker for disease diagnosis in various domestic animals. The breed-dependent variation in expression of miRNAs and polymorphism in miRNA target sites on important genes related with animal productivity could be implemented as a basis in selection programs in livestock species.

\section{Acknowledgement}

This study was supported by the ICAR-Indian Veterinary Research Institute, Government of India. The authors would also wish to acknowledge the Director and Joint Director Academic and Research, IVRI, Bareilly, India, for their kind support to carry out this review.

\section{References}

Basagoudanavar, S H, Hosamani M, Selvan R T, Sreenivasa B P, Sanyal A and Venkataramanan R. 2018. Host serum microRNA profiling during the early stage of foot-and-mouth disease virus infection. Archives of Virology 3:1-9.

Caiment, F, Charlier C, Hadfield T, Cockett N, Georges M and Baurain D. 2010. Assessing the effect of the CLPG mutation on the microRNA catalog of skeletal muscle using high-throughput sequencing. Genome Research 13.

Castoldi, M, Benes V, Hentze M W and Muckenthaler M U. 2007. miChip: a microarray platform for expression profiling of microRNAs based on locked nucleic acid (LNA) oligonucleotide capture probes. Methods 43(2): 146-52.

Chen, C, Deng B, Qiao M, Zheng R, Chai J, Ding Y, Peng J and Jiang S. 2012. Solexa sequencing identification of conserved and novel microRNAs in backfat of Large White and Chinese Meishan pigs. PloS One 7(2):31426.

Chen, C, Ridzon D A, Broomer A J, Zhou Z, Lee D H, Nguyen J T, Barbisin M, Xu N L, Mahuvakar V R, Andersen M R and Lao K Q. 2005. Real-time quantification of microRNAs by stem-loop RT-PCR. Nucleic Acids Research 33(20): 179.

Cho, I S, Kim J, Seo H Y, Lim D H, Hong J S, Park Y H, Park D C, Hong K C, Whang K Y and Lee Y S. 2010. Cloning and characterization of microRNAs from porcine skeletal muscle and adipose tissue. Molecular Biology Reports 37(7):3567-74.

Cissell, K A, Rahimi Y, Shrestha S, Hunt E A and Deo S K. 2008. Bioluminescencebased detection of microRNA, miR21 in breast cancer cells. Anal Chem 80(7):2319-25. 
Clop, A, Marcq F, Takeda H, Pirottin D, Tordoir X, Bibé B, Bouix J, Caiment F, Elsen JM, Eychenne F and Larzul C. 2006. A mutation creating a potential illegitimate microRNA target site in the myostatin gene affects muscularity in sheep. Nature Genetics 38(7):813.

Condorelli G, Latronico M V and Cavarretta E. 2014. microRNAs in cardiovascular diseases: current knowledge and the road ahead. Journal of the American College of Cardiology 63(21):217787.

Cooper, D K, Ezzelarab M B, Hara H, Iwase $\mathrm{H}$, Lee $\mathrm{W}$, Wijkstrom $\mathrm{M}$ and Bottino R. 2016. The pathobiology of pig-to-primate xenotransplantation: a historical review. Xenotransplantation 23(2):83-105.

dePlanell-Saguer M and Rodicio M C. 2011. Analytical aspects of microRNA in diagnostics: a review. Analytica chimica acta 699(2):134-52.

Dilda, F, Gioia G, Pisani L, Restelli L, Lecchi C, Albonico F, Bronzo V, Mortarino $\mathrm{M}$ and Ceciliani F. 2012. Escherichia coli lipopolysaccharides and Staphylococcus aureus enterotoxin B differentially modulate inflammatory microRNAs in bovine monocytes. The Veterinary Journal 192(3):514-6.

Dirksen $\mathrm{K}$, Verzijl $\mathrm{T}$, van den Ingh $\mathrm{T} \mathrm{S}$, Vernooij $\mathrm{J} C$, van der Laan $\mathrm{L} J$, Burgener I A, Spee B and Fieten H. 2016. Hepatocyte-derived microRNAs as sensitive serum biomarkers of hepatocellular injury in Labrador retrievers. The Veterinary Journal 211:75-81.

Dong, H, Gao Q, Peng X, Sun Y, Han T, Zhao B, Liu Y, Wang C, Song X, Wu J and Yang L. 2017. Circulating MicroRNAs as potential biomarkers for veterinary infectious diseases. Frontiers in veterinary science 4:186.
Driskell J D, Seto A G, Jones L P, Jokela S, Dluhy R A, Zhao Y P and Tripp R A. 2008. Rapid microRNA (miRNA) detection and classification via surface-enhanced Raman spectroscopy (SERS). Biosensors and Bioelectronics 24(4):917-22.

Fang, S, Lee H J, Wark A W and Corn R M. 2006. Attomole microarray detection of microRNAs by nanoparticleamplified SPR imaging measurements of surface polyadenylation reactions. Journal of the American Chemical Society 128(43):14044-6.

Fatima A and Morris DG. 2013. MicroRNAs in domestic livestock. Physiological Genomics 45(16):685-96.

Friedman R C, Farh K K, Burge C B and Bartel D P. 2009. Most mammalian mRNAs are conserved targets of microRNAs. Genome Research 19(1):92-105.

Fujiwara-Igarashi, A, Igarashi H, Mizutani N, Goto-Koshino Y, Takahashi M, Ohno $\mathrm{K}$ and Tsujimoto H. 2015. Expression profile of circulating serum microRNAs in dogs with lymphoma. The Veterinary Journal 205(2):31721.

Galio, L, Droineau S, Yeboah P, Boudiaf H, Bouet S, Truchet S and Devinoy E. 2012. MicroRNA in the ovine mammary gland during early pregnancy: spatial and temporal expression of miR-21, miR-205, and miR-200. Physiological Genomics 45(4):151-61.

Geiss, G K, Bumgarner R E, Birditt B, Dahl T, Dowidar N, Dunaway D L, Fell H P, Ferree S, George R D, Grogan T and James J J. 2008. Direct multiplexed measurement of gene expression with color-coded probe pairs. Nature Biotechnology 26(3):317.

Govindaraju, A, Uzun A, Robertson L, Atli M 
O, Kaya A, Topper E, Crate E A, Padbury J, Perkins A and Memili E. 2012. Dynamics of microRNAs in bull spermatozoa. Reproductive Biology and Endocrinology 10(1):82.

Gustafson, D, Tyryshkin K and Renwick N. 2016. microRNA-guided diagnostics in clinical samples. Best practice \& research Clinical endocrinology \& metabolism 30(5):563-75.

Gutkoska, J, LaRocco M, Ramirez-Medina E, De Los Santos $\mathrm{T}$ and Lawrence $\mathrm{P}$. 2017. Host microRNA-203a is antagonistic to the progression of footand-mouth disease virus infection. Virology. 504:52-62.

Hafner, M, Renwick N, Farazi T A, Mihailović A, Pena J T and Tuschl T. 2012. Barcoded cDNA library preparation for small RNA profiling by next-generation sequencing. Methods 58(2):164-70.

Hansen, E P, Kringel H, Thamsborg S M, Jex $A$ and Nejsum P. 2016. Profiling circulating miRNAs in serum from pigs infected with the porcine whipworm, Trichuris suis. Veterinary parasitology 223:30-3.

Holley, C L and Topkara V K. 2011. An introduction to small non-coding RNAs: miRNA and snoRNA. Cardiovascular drugs and therapy 25(2):151.

Hulanicka, M, Garncarz M, ParzenieckaJaworska M and Jank M. 2014. Plasma miRNAs as potential biomarkers of chronic degenerative valvular disease in Dachshunds. BMC Veterinary Research 10(1):205.

Ioannidis, J and Donadeu F X. 2017. Changes in circulating microRNA levels can be identified as early as day 8 of pregnancy in cattle. PloS one 12(4):0174892.

Ji Z, Wang G, Xie Z, Wang J, Zhang C, Dong $F$ and Chen C. 2012. Identification of novel and differentially expressed microRNAs of dairy goat mammary gland tissues using Solexa sequencing and bioinformatics. PloS one. 7(11):49463.

Jin W, Ibeagha-Awemu E M, Liang G, Beaudoin F and Zhao X. 2014. Transcriptome microRNA profiling of bovine mammary epithelial cells challenged with Escherichia coli or Staphylococcus aureus bacteria reveals pathogen directed microRNA expression profiles. BMC genomics 15(1):181.

Lawless, N, Foroushani A B, McCabe M S, O'Farrelly C and Lynn D J. 2013. Next generation sequencing reveals the expression of a unique miRNA profile in response to a gram-positive bacterial infection. PloS one. 8(3):57543.

Lawless N, Reinhardt T A, Bryan K, Baker M, Pesch B, Zimmerman D, Zuelke K, Sonstegard T, O'farrelly C, Lippolis J D and Lynn D J. 2014. MicroRNA regulation of bovine monocyte inflammatory and metabolic networks in an in vivo infection model. G3: Genes, Genomes, Genetics g3-113.

Li G, Li Y, Li X, Ning X, Li M and Yang G. 2011. MicroRNA identity and abundance in developing swine adipose tissue as determined by Solexa sequencing. Journal of cellular biochemistry 112(5):1318-28.

Li, H, Li S, Yu B and Liu S. 2012. Expression of miR-133 and miR-30 in chronic atrial fibrillation in canines. Molecular Medicine Reports 5(6):1457-60.

Li, Z, Lan X, Guo W, Sun J, Huang Y, Wang J, Huang T, Lei C, Fang $X$ and Chen H. 2012. Comparative transcriptome profiling of dairy goat microRNAs from dry period and peak lactation mammary gland tissues. PloS One 7(12):52388. 
Malvisi, M, Palazzo F, Morandi N, Lazzari B, Williams $J$ L, Pagnacco $G$ and Minozzi G. 2016. Responses of bovine innate immunity to Mycobacterium avium subsp. paratuberculosis infection revealed by changes in gene expression and levels of microRNA. PLoS One 11(10):0164461.

Markkandan K, Ahn K, Lee D J, Kim T I, Dang C, Hong S E, Yoon H B, Lim H $\mathrm{J}$ and Hong $\mathrm{C}$ P. Profiling and identification of pregnancy-associated circulating microRNAs in dairy cattle. Genes \& Genomics 1-7.

McDaneld, T G, Smith T P, Doumit M E, Miles J R, Coutinho L L, Sonstegard T S, Matukumalli L K, Nonneman D J and Wiedmann R T. 2009. MicroRNA transcriptome profiles during swine skeletal muscle development. BMC genomics 10(1):77.

Naeem A, Zhong K, Moisá S J, Drackley J K, Moyes K M and Loor J J. 2012. Bioinformatics analysis of microRNA and putative target genes in bovine mammary tissue infected with Streptococcus uberis1. Journal of dairy science 95(11):6397-408.

Nelson, P T, Baldwin D A, Kloosterman W P, Kauppinen S, Plasterk $\mathrm{R} \mathrm{H}$ and Mourelatos Z. 2006. RAKE and LNAISH reveal microRNA expression and localization in archival human brain. RNA 12(2):187-91.

Nielsen M, Hansen J H, Hedegaard J, Nielsen $\mathrm{R} \mathrm{O}$, Panitz $\mathrm{F}$, Bendixen $\mathrm{C}$ and Thomsen B. 2010. MicroRNA identity and abundance in porcine skeletal muscles determined by deep sequencing. Animal Genetics 41(2):159-68.

Ogorevc, J, Kunej T, Razpet A and Dovc P. 2009. Database of cattle candidate genes and genetic markers for milk production and mastitis. Animal Genetics 40(6):832-51.
Pandey, A, Sahu A R, Wani S A, Saxena S, Kanchan S, Sah V, Rajak K K, Khanduri A, Sahoo A P, Tiwari A K and Mishra B. 2017. Modulation of Host miRNAs Transcriptome in Lung and Spleen of Peste des Petits Ruminants Virus Infected Sheep and Goats. Frontiers in microbiology 8:1146.

Qi X, Wang T, Xue Q, Li Z, Yang B and Wang J. 2018. MicroRNA expression profiling of goat peripheral blood mononuclear cells in response to peste des petits ruminants virus infection. Veterinary Research 49(1):62.

Roepstorff, A, Mejer H, Nejsum $\mathrm{P}$ and Thamsborg S M. 2011. Helminth parasites in pigs: new challenges in pig production and current research highlights. Veterinary Parasitology 180(1-2):72-81.

Shan J, Feng L, Luo L, Wu W, Li C, Li S and Li Y. 2011. MicroRNAs: potential biomarker in organ transplantation. Transplantation Immunology 24(4):210-5.

Shaughnessy, R G, Farrell D, Riepema K, Bakker D and Gordon S V. 2015. Analysis of bio banked serum from a Mycobacterium avium subsp paratuberculosis bovine infection model confirms the remarkable stability of circulating miRNA profiles and defines a bovine serum miRNA repertoire. PLoS One 10(12):145089.

Stenfeldt, C, Arzt J, Smoliga G, LaRocco M, Gutkoska J and Lawrence P. 2017. Proof-of-concept study: profile of circulating microRNAs in Bovine serum harvested during acute and persistent FMDV infection. Virology journal 14(1):71.

Sun, J, Aswath K, Schroeder S G, Lippolis J $\mathrm{D}$, Reinhardt T A and Sonstegard T S. 2015. MicroRNA expression profiles of bovine milk exosomes in response 
to Staphylococcus aureus infection. BMC genomics 16(1):806.

Tao, J, Yang X, Han Z, Lu P, Wang J, Liu X, Wu B, Wang Z, Huang Z, Lu Q and Tan R. 2015. Serum MicroRNA-99a helps detect acute rejection in renal transplantation. Transplant Proc. Elsevier 47(6):1683-1687.

Taxis T M, Bauermann F V, Ridpath J F and Casas E. 2017. Circulating microRNAs in serum from cattle challenged with bovine viral diarrhea virus. Frontiers in genetics 8:91.

Tesfaye, D, Worku D, Rings F, Phatsara C, Tholen E, Schellander K and Hoelker M. 2009. Identification and expression profiling of microRNAs during bovine oocyte maturation using heterologous approach. Molecular reproduction and development 76(7):665-77.

Topkara, V K and Mann D L. 2011. Role of microRNAs in cardiac remodeling and heart failure. Cardiovascular drugs and therapy 25(2):171-82.

Wang, J, Chen J and Sen S. 2016. MicroRNA as biomarkers and diagnostics. Journal of cellular physiology 231(1):25-30.

Watts, J L. 1988. Etiological agents of bovine mastitis. Veterinary Microbiology 16(1):41-66.

Weber, K, Rostert N, Bauersachs S and Wess G. 2015. Serum microRNA profiles in cats with hypertrophic cardiomyopathy. Molecular and Cellular Biochemistry 402(1-2):17180.

Zhang S, Zhao F, Wei C, Sheng X, Ren H, Xu $\mathrm{L}$, Lu J, Liu J, Zhang L and Du L. 2013. Identification and characterization of the miRNA transcriptome of Ovis aries. PLoS One 8(3):58905.

Zhang, Y, Zheng S, Geng Y, Xue J, Wang Z, Xie $\mathrm{X}$, Wang J, Zhang $\mathrm{S}$ and Hou $\mathrm{Y}$. 2015. MicroRNA profiling of atrial fibrillation in canines: miR-206 modulates intrinsic cardiac autonomic nerve remodeling by regulating SOD1. PloS one 10(3):122674.

Zhou, P, Zhang X, Zeng W, Zheng Q, Hao X, Lin X, Zheng Y, Wang L, Zhang G and Li S. 2017. MicroRNA expression analysis of feline and canine parvovirus infection in vivo (felis). PloS one 12(10):185698.

\section{How to cite this article:}

Aamir Bashir Wara, Supriya Chhotaray, Burhan Ud Din Shafi, Snehasmita Panda, Mitek Tarang, and Amit Kumar. 2019. Role of miRNA Signatures in Health and Productivity of Livestock. Int.J.Curr.Microbiol.App.Sci. 8(04): 727-738. doi: https://doi.org/10.20546/ijcmas.2019.804.079 